\title{
Recent Viewpoints on the Thermodynamics of Fluctuationless First-Order Phase Transitions
}

\author{
J. Ortín and J. Goicoechea \\ Departament ECM, Facultat de Física, Universitat de Barcelona, Diagonal 647, 08028 Barcelona, \\ Catalonia, Spain
}

\begin{abstract}
This work focuses on the macroscopic behaviour of a large family of first-order phase transitions, including thermoelastic martensitic transformations, which are practically insensitive to thermal fluctuations and need to be driven by an external field. The Random Field Ising Model at $T=0$ is used to carry out a detailed study of the dynamics and thermodynamics of this family of fluctuationless transitions. The main difficulties expected for performing the same kind of analysis on martensitic transformations are outlined.
\end{abstract}

\section{INTRODUCTION}

A thermodynamic study of the martensitic transition (MT) is particularly appealing because the MT is very sensitive to both temperature and applied stress. On one side, the thermodynamics of MT is simplified by the fact that the transition is difusionless: the material behaves thermodynamically as a single component system. In addition, the transition is perfectly reversible in many systems, in the sense that the original phase is recovered nearly exactly after a complete transformation cycle. As a consequence, thermodynamic magnitudes that are state functions recover their original values after the cycle, a property that can be succesfully exploited to derive a number of thermodynamic relations. On the side of the difficulties we have, first, the important role of crystalline defects (present originally in the material and generated in the transition), manifested on the heterogeneous character of nucleation and growth. Second, the presence of a complex elastic energy field in the material, as a result of the defects themselves and the shape and volume incompatibilities between the two crystal lattices. Because of these two ingredients, the internal energy of the system can be presumed to be highly heterogeneous in space. This spatial complexity is responsible for the dissipation of energy in the transition, whose macroscopic manifestation is the fact that a complete MT cycle displays hysteresis: from a thermodynamic point of view, the MT is an irreversible process. To stablish a proper thermodynamic approach of the MT, including the right energy balance at the transition, the presence of heterogeneities and irreversibilities must be taken into account from the beginning. Unfortunately, such an scheme has not been definitely stablished yet.

The last two decades have seen different approaches to the subject [1] which, in many instances, have been found to be conflicting. It is not our purpose here to contribute to the polemics with our own point of view. Instead, we will take the opportunity to present the subject from an entirely new perspective, emerged from the seminal work of Sethna et al. [2] on the hysteresis of fluctuationless, first-order phase transitions. From this point of view, the kinetic and thermodynamic behaviour of a thermoelastic MT is typical of first-order phase transitions for which thermal fluctuations are practically irrelevant, and therefore require an external field (here temperature or applied stress) to be driven. Indeed, upon driving a martensitic system through the $\mathrm{MT}$, it follows a sequence of metastable two-phase configurations. Each configuration is a relative energy minimum in which the 
system is trapped by the building up of elastic strain energy. The transformation resumes when the driving field modifies the energy landscape to a point such that the configuration becomes unstable again, an avalanche of non-equilibrium transformation events takes place, and a new metastable configuration is reached. This picture of the MT is supported by several observations: (i) The duration of a tranformation event (an avalanche) is orders of magnitude smaller than the time between events. (ii) The transformation events are dissipative processes, and transformation-retransformation trajectories form hysteresis cycles. (iii) The transformation is athermal: it only progresses with the help of a driving field (stress or temperature). (iv) Often the transformation trajectories are highly reproducible from cycle to cycle; an extreme example of this reproducibility is the return-point memory, observed repeatedly in thermoelastic MT.

The paper is organized in the following way: Section 2 focuses on the Random Field Ising Model (RFIM) driven by a magnetic field in the absence of thermal fluctuations. The RFIM, formulated in the language of magnetic systems, can be regarded as the paradigm of fluctuationless first-order phase transitions. We explain how the model captures the essential physics of hysteresis and metastability in the systems considered, and the dependence of the transformation trajectories on previous history. We develop the thermodynamics and the energy balance of the model at the phase transition. Section 3 summarizes the difficulties to be faced when trying to translate ideas and results from the RFIM to thermoelastic MT.

\section{THERMODYNAMICS AND KINETICS OF THE RFIM AT $T=0$}

In Statistical Mechanics [3], first-order phase transitions are usually described in terms of the Ising model driven by an external magnetic field $H$. The system is represented by a periodic lattice in $d$ dimensions. On each lattice site there is a magnetic moment (or spin) $s_{i}$, which can take two values: +1 if $s_{i}$ points to the positive direction of $H,-1$ if it points to the opposite direction. The Hamiltonian of the model is given by:

$$
\mathcal{H}=-J \sum_{<i j>} s_{i} s_{j}-H \sum_{i} s_{i}
$$

The first term represents an interaction of every spin with its $\gamma$ nearest neighbours. The interaction has strength $J>0$ (ferromagnetic): it favours that all spins align in the same direction. The summation extends over all couples $i, j$ of nearest neighbours (represented by $<i j>$ ). The second term represents the interaction of each spin with the external field $H$, which tends to align the spin in the direction of $H$. The summation now extends over the $N$ lattice sites. The energy of the system, given by the Hamiltonian, depends simultaneously on the external field $H$ and the particular configuration of the spins $\left\{s_{i}\right\}$. Note also that $\Sigma_{i} s_{i}$ is the total magnetization of the system, $M$, to which the external field couples. At $T=0$ (i.e. in the absence of thermal fluctuations of the spin orientations) it is not difficult to see that the configuration $\{-1\}$ (all the spins oriented in the direction of negative $H$ ) absolutely minimizes the energy for $-\infty<H \leq 0$, while $\{+1\}$ does the same for $0 \leq H<+\infty$. For $H=0$ the energy of both configurations is the same: at this point the phase transition between these two configurations takes place in equilibrium. In the absence of thermal fluctuations, however, a spin (or a number of spins) from $\{-1\}$ that attempts to revert its orientation experiences an energy barrier against the flip that does not disappear until:

$$
\frac{\partial \mathcal{H}}{\partial s_{i}}=0 \quad \rightarrow \quad-J \sum_{<j|i\rangle} s_{j}-H=0 \quad \rightarrow \quad H=\gamma J .
$$

At this value of $H$, any spin of the configuration $\{-1\}$ flips without opposition from -1 to +1 and subsequently, due to the interaction with its neighbours, triggers an avalanche of spin flips that 
spreads to the entire system. The system reaches the new configuration $\{+1\}$. Equivalently, in the reverse direction, a spin of the configuration $\{+1\}$ experiences an energy barrier against flipping until:

$$
\frac{\partial \mathcal{H}}{\partial s_{i}}=\dot{0} \rightarrow-J \sum_{<j|i\rangle} s_{j}-H=0 \quad \rightarrow \quad H=-\gamma J .
$$

If we make a plot of the magnetization against the magnetic field, we observe a rectangular hysteresis cycle: rising the field from $-\infty$, the magnetization is $M=-N$ while $-\infty<H \leq \gamma J$, changes suddenly to $M=+N$ at $H=\gamma J$, and remains there for $H>\gamma J$. Lowering the field from $+\infty$, the magnetization is $M=+N$ while $-\gamma J \leq H<+\infty$, changes suddenly to $M=-N$ at $H=-\gamma J$, and remains there for $H<-\gamma J$. The area inside the cycle is $(2 \gamma J)(2 N)$ : this is effectively the energy excess input into the system (in comparison to the equilibrium transition) as a consequence of having scanned $H$ until the limits of metastability of the system. This energy excess is released irreversibly in the two avalanches in which the magnetization changes abruptly.

The model described by Eq.(1) represents a homogeneous system, whose properties do not depend on the lattice site we are looking at. Heterogeneities (originating from the presence of quenched defects or other forms of static disorder) can be incorporated to the model, basically, in two ways: (i) Making the interaction between spins to depend on lattice site, so that the interaction term reads now $\sum_{<i j>} J_{i j} s_{i} s_{j}$; the resulting model is known as Random Bond Ising Model (RBIM) [4]. (ii) Placing a static magnetic field $h_{i}$, taken from a random distribution, on each lattice site; the Hamiltonian of this model, known as Random Field Ising Model (RFIM), is given by:

$$
\mathcal{H}=-J \sum_{<i j\rangle} s_{i} s_{j}-\sum_{i} h_{i} s_{i}-H \sum_{i} s_{i}
$$

For convenience, the values $\left\{h_{i}\right\}$ are taken from a gaussian distribution of width $\sigma$ and centered around 0 , thus having the statistical properties of the disorder characterized by this single parameter $\sigma$. The RFIM was introduced in the context of systems with hysteresis by Sethna and collaborators [2], and we will adopt it here as the emblematic model for this kind of problems.

\subsection{Dynamics of the RFIM}

Once a (static) realization of random fields $\left\{h_{i}\right\}$ is selected, the evolution of the system is dictated by the behaviour of $\mathcal{H}$, which depends on the configuration $\left\{s_{i}\right\}$ and the field $H$. Hence, formally, the evolution can be described by:

$$
d \mathcal{H}=\sum_{i}\left(\frac{\partial \mathcal{H}}{\partial s_{i}}\right) d s_{i}+\left(\frac{\partial \mathcal{H}}{\partial H}\right) d H
$$

which results in:

$$
d \mathcal{H}=-\sum_{i}\left(J \sum_{<j \mid i>} s_{j}+h_{i}+H\right) d \dot{s}_{i}-\sum_{i} s_{i} d H
$$

The term between brackets is the local magnetic field, $F_{i}$, experienced by the spin $s_{i}$. Then:

$$
d \mathcal{H}=-\sum_{i} F_{i} d s_{i}-\sum_{i} s_{i} d H
$$

This equation is very instructive. To see why, let us consider the evolution of the system from a value $H \ll h_{i}$ for all lattice sites, for which the system adopts the configuration $\{-1\}$. The local fields $F_{i}$ are all negative and hence any change $d s_{i}>0$ is forbidden, in the absence of thermal fluctuations, because it contributes to increase the energy. As we increase $H$, at constant $\left\{s_{i}\right\}, \mathcal{H}$ increases as a 


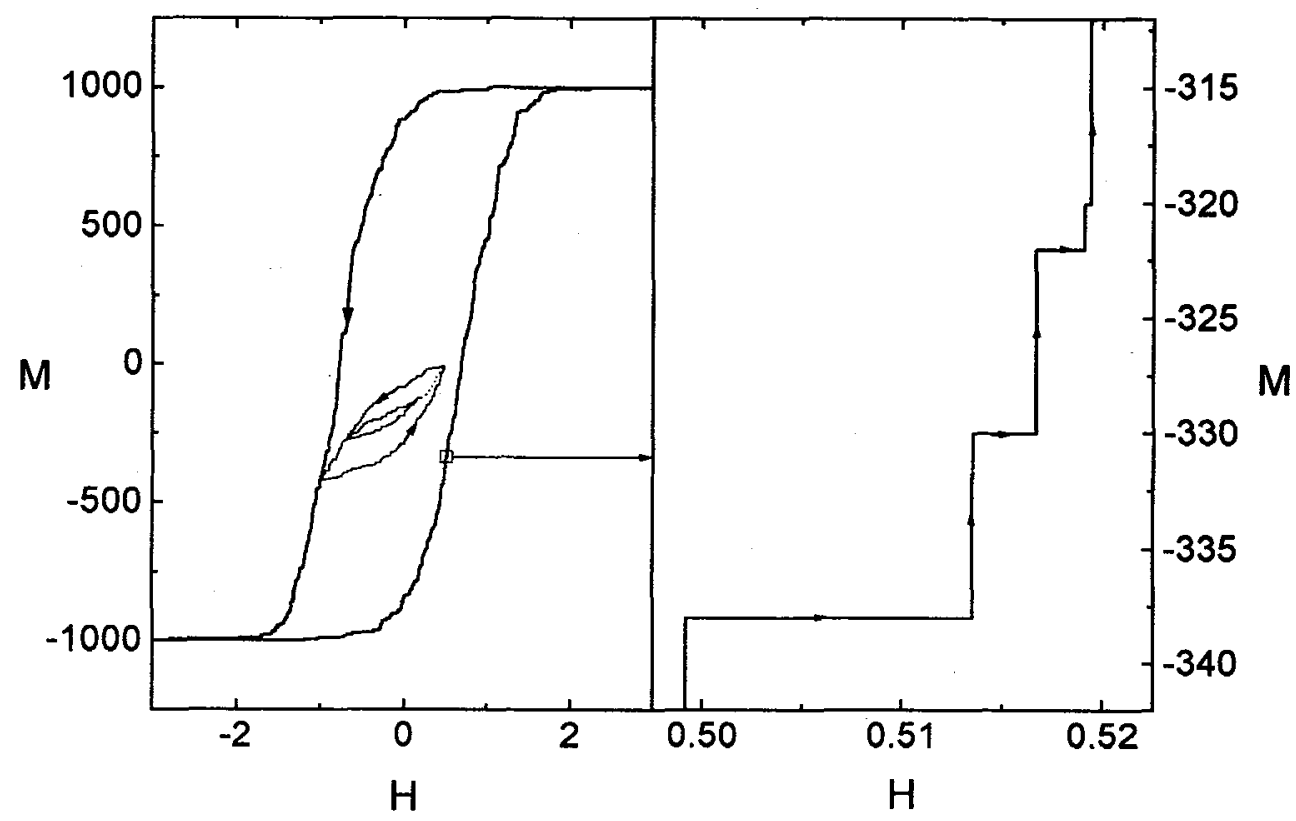

Figure 1: On the left: transformation-retransformation trajectories of a RFIM with $d=1, N=1000$ spins and $\sigma=1$, including two partial cycles to demonstrate the return-point memory property. On the right: a magnified portion of the ascending trajectory, showing a staircase structure.

result of the coupling between $H$ and the magnetization $\sum_{i} s_{i}$. At the same time the $F_{i}$ increase as well, until $F_{i}=0$ in a first lattice site for some value of $H$. This is the minimum condition for a spin $s_{i}$ to flip $\left(d s_{i}>0\right)$ at given $H(d H=0)$ without energy barriers $(d \mathcal{H}=0)$. But the flip of $s_{i}$ modifies the local field $F_{j}$ experienced by the neighbours. One or more $F_{j}$ may become positive, making the corresponding spins $j$ to flip. On their turn, these spins may trigger new flips of the neighbours, and so on, giving rise to an avalanche of spin flips at constant magnetic field. The avalanche, and the concomitant abrupt jump in the magnetization of the system, arrests when no other spins experience a positive local field. Note that, although the first spin flips in equilibrium $(d \mathcal{H}=0)$, all others spins in the avalanche flip out of equilibrium $(d \mathcal{H}<0)$. From this situation, let us increase $H$ again monotonously, until a new spin -1 experiences a local field $F_{i}=0$ and flips in equilibrium (and eventually, triggers a new avalanche of non-equilibrium spin flips at constant $H$ ), increase $H$ again and so on, until the system finally reaches the configuration $\{+1\}$. From here, upon decreasing the applied field, the reverse process from $\{+1\}$ to $\{-1\}$ is equivalent to the process just described. The trajectory followed by the system in configuration space is different, however, because avalanches are irreversible. Figure 1 shows an example of the resulting $M-H$ cycle, a set of partial trajectories inside the cycle, and a detail of the staircase structure of a transformation trajectory.

We emphasize two properties of the dynamics. First, it is completely deterministic: given a realization $\left\{h_{i}\right\}$, the trajectories followed in configuration space are perfectly reproducible. Second, it is adiabatic: the evolution is independent of the rate at which the system is driven. This property corresponds to a complete separation of time scales: the avalanches are instantaneous on the time scale of variation of the external field. 


\subsection{Memory properties of partial transformation trajectories}

Consider the partial trajectories shown in Figure 1: every time that $H$ returns to its original value, having completed a (partial) cycle, the system returns to its original configuration. This memory property holds for all partial cycles (including cycles inside cycles), giving rise to a complex hierarchical structure of transformation trajectories. Remember that this behaviour, common to many ferromagnetic materials [5], has been repeatedly observed also in the MT, either stress-induced or thermally-induced [6], and in a variety of other systems [7].

The physical origin of the memory of partial transformation trajectories is clearly understood in the framework of the fluctuationless RFIM [2]. Note in first place that the possible configurations of the system admit a partial ordering:

$$
\vec{s}=\left\{s_{1}, \ldots, s_{N}\right\} \geq \vec{r}=\left\{r_{1}, \ldots, r_{N}\right\} \quad \Longleftrightarrow \quad s_{i} \geq r_{i} \quad \forall i
$$

Although most configurations are not mutually ordered, this property is useful because, in second place, it can be shown that the dynamics preserves the partial ordering. Suppose that $\vec{s}(t)$ evolves under $H_{s}(t)$ and $\vec{r}(t)$ evolves under $H_{r}(t)$. Then:

$$
\left.\begin{array}{c}
\vec{s}(0) \geq \vec{r}(0) \\
H_{s}(t) \geq H_{r}(t)
\end{array}\right\} \Rightarrow \vec{s}(t) \geq \vec{r}(t)
$$

To prove it, remember that a spin flips when its local field $F_{i}$ changes sign. For $\vec{s}<\vec{r}$ there should be a first time $t$ such that $F_{i}^{s}<F_{i}^{r}$. This is not possible, since $h_{i}^{s}=h_{i}^{r}, H_{s} \geq H_{r}$ and the neighbours of the spin considered are $s_{j} \geq r_{j}$. In third place, a monotonous evolution of $H$ takes the system through a single sequence of configurations because the dynamics is adiabatic.

The memory properties of partial cycles are a consequence of the partial ordering of the configurations, the preservation of the ordering by the dynamics, and the adiabaticity. Suppose that, from a given configuration $\vec{s}(0)$, we let the field evolve during a time $\Delta t$ between an initial minimum value $H_{A}$ and a final maximum value $H_{B}$. The evolution may be non-monotonic and generate partial cycles. Even so, we can prove that the final configuration $\vec{s}(t)$ is the same that we would have reached if the field had evolved monotonously between $H_{A}$ and $H_{B}$. To see it, consider two hypothetical monotonous evolutions of the field between $H_{A}$ and $H_{B}$. In the first one, $H_{\text {min }}(t)$, the field remains always equal or smaller than $H(t)$. In the second one, $H_{\max }(t)$, it keeps always equal or larger than $H(t)$. Since the dynamics preserves the partial ordering of configurations, we have at all times $\vec{s}_{\text {min }}(t) \leq \vec{s}(t)$ and $\vec{s}(t) \leq \vec{s}_{\max }(t)$. But, at the same time, since the two evolutions $H_{m i n}(t)$ and $H_{m a x}(t)$ are monotonic, we start from the same configuration $\vec{s}(0)$, and the dynamics is adiabatic, it turns out that $\vec{s}_{\min }(\Delta t)=\vec{s}_{\max }(\Delta t)$. In conclusion, $\vec{s}_{\min }(\Delta t)=\vec{s}(\Delta t)=\vec{s}_{\max }(\Delta t)$.

\subsection{Energy balance}

The Hamiltonian of the RFIM, given by Eq.(4), represents the energy of the spin system in a configuration $\left\{s_{i}\right\}$ in the presence of an external magnetic field $H$. Each term of $\mathcal{H}$ accounts for a different contribution: the first two terms account for the energy stored in the system as a consequence of the spin-spin interaction (first term) and the interaction between spins and quenched disorder (second term). The third one accounts for the energy input into the system as a result of the interaction between the spins and the external magnetic field.

Eq.(7) states that changes in $\mathcal{H}$ arise from two distinct mechanisms. Let us consider first the horizontal segments of the $M-H$ curve in Figure 1. There, $d\left\{s_{i}\right\}=0$ (the spin configuration remains 
unchanged) and we have:

$$
\left.d \mathcal{H}\right|_{\left\{s_{i}\right\}}=-\sum_{i} s_{i} d H=-M d H
$$

The change in $\mathcal{H}$ is caused by the variation of the external field. Consider now the vertical segments of the $M-H$ curve, where $d H=0$. We have there:

$$
\left.d \mathcal{H}\right|_{H}=-\sum_{i} F_{i} d s_{i}
$$

Remember that, according to the dynamics, the first spin to flip in an avalanche flips with $F_{i}=0$ and hence does not produce a change of $\mathcal{H}$; this spin flips in equilibrium, in a reversible way. If the first flip triggers new spin flips, however, these new flips take place with $F_{i} \neq 0$ (out of equilibrium) and therefore produce changes in $\mathcal{H}$. It is not difficult to see that $F_{i}$ and $d s_{i}$ have the same sign (positive when the spin flips from -1 to +1 , negative in the opposite sense) and hence $\left.d \mathcal{H}\right|_{H}<0$ in all cases. We conclude that magnetization changes involving more than one spin (avalanches) are intrinsically dissipative processes. The energy dissipation at avalanches is the physical origin of hysteresis.

Next, we can carry out a detailed analysis of the energy balance in the system. In very general terms, a magnetic system whose thermodynamic evolution is quasi-static and dissipative verifies:

$$
d U=\vec{d} W+\vec{d} Q=H d M+T \vec{d} S_{e}
$$

where $\vec{d} S_{e}$ is the entropy flow into the system. In general, also:

$$
\vec{a} S_{e}+\vec{a} S_{i}=d S
$$

which states the fact that changes in the entropy of the system may have two origins: the flow $d S_{e}$ from outside, and the entropy production $d S_{i}$, inside the system, due to the presence of irreversibilities. We have then:

$$
d U=H d M+T d S-T d S_{i}
$$

which is the fundamental thermodynamic identity for a magnetic system. Immediately, for a closed cycle at constant temperature:

$$
\oint \vec{d} S_{i}=\frac{1}{T} \oint H d M
$$

which relates directly the entropy production in the hysteresis cycle with the area enclosed by the cycle. Note that the second law of thermodynamics dictates $d S_{i} \geq 0$, and hence Eq.(15) determines the only possible direction of the $M-H$ cycle.

The Hamiltonian of the RFIM and the internal energy of the system at constant temperature are related by the Legendre transformation:

$$
\mathcal{H}=U-T S-H M=\mathcal{F}-H M,
$$

where $\mathcal{F}$ is the Helmholtz free energy. Hence, at constant temperature:

$$
\left.d \mathcal{H}\right|_{T}=-T \vec{d} S_{i}-M d H
$$

Comparing to $d \mathcal{H}$, given by Eq.(7), we arrive to:

$$
T d S_{i}=\sum_{i} F_{i} d s_{i}
$$

We have accomplished one of the primary goals of the Thermodynamics of Irreversible Processes: to identify, the entropy production. This result fits nicely in our picture that the energy dissipation takes place at the avalanches $\left(F_{i} \neq 0\right)$. 


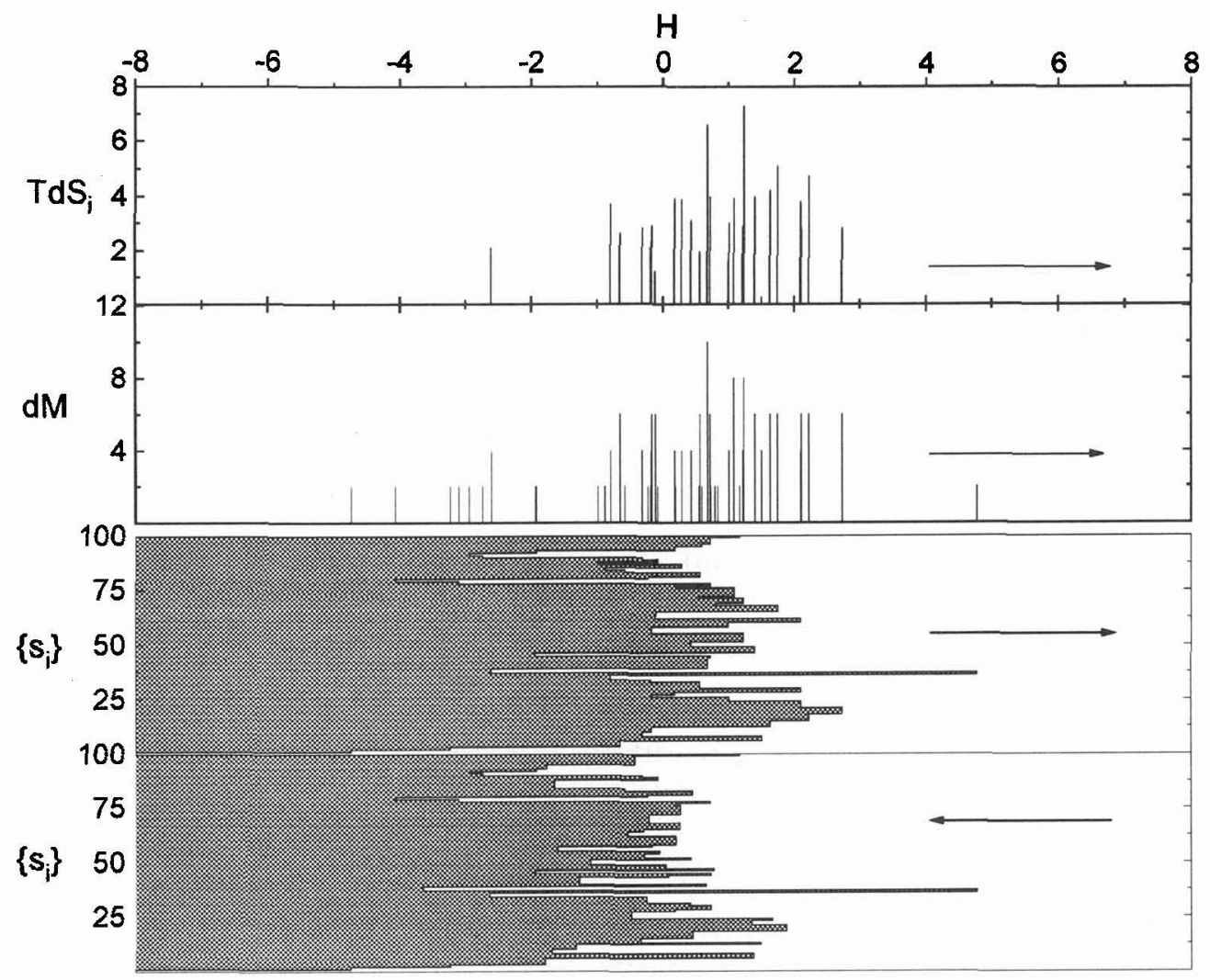

Figure 2: From top to bottom: energy dissipation, magnetization jumps, and sequence of configurations followed on rising the field, and sequence of configurations followed on lowering the field, in a RFIM with $d=1, N=100$ and $\sigma=5$. Gray lines represent spins -1 and white lines spins +1 .

In addition, we may go one step further and separate:

$$
\sum_{i} F_{i} d s_{i}=\sum_{i}\left(J \sum_{<j \mid i>} s_{j}+h_{i}\right) d s_{i}+H \sum_{i} d s_{i}
$$

which corresponds to:

$$
T d S_{i}=-d \mathcal{F}+H d M
$$

or, equivalently:

$$
H d M=d \mathcal{F}+T \tilde{d} S_{i}
$$

This equation should be read as follows: the energy input by the external field into the system is partially stored in the system and partially dissipated. The energy stored is given by:

$$
d \mathcal{F}=-\sum_{i}\left(J \sum_{<j \mid i>} s_{j}+h_{i}\right) d s_{i}
$$




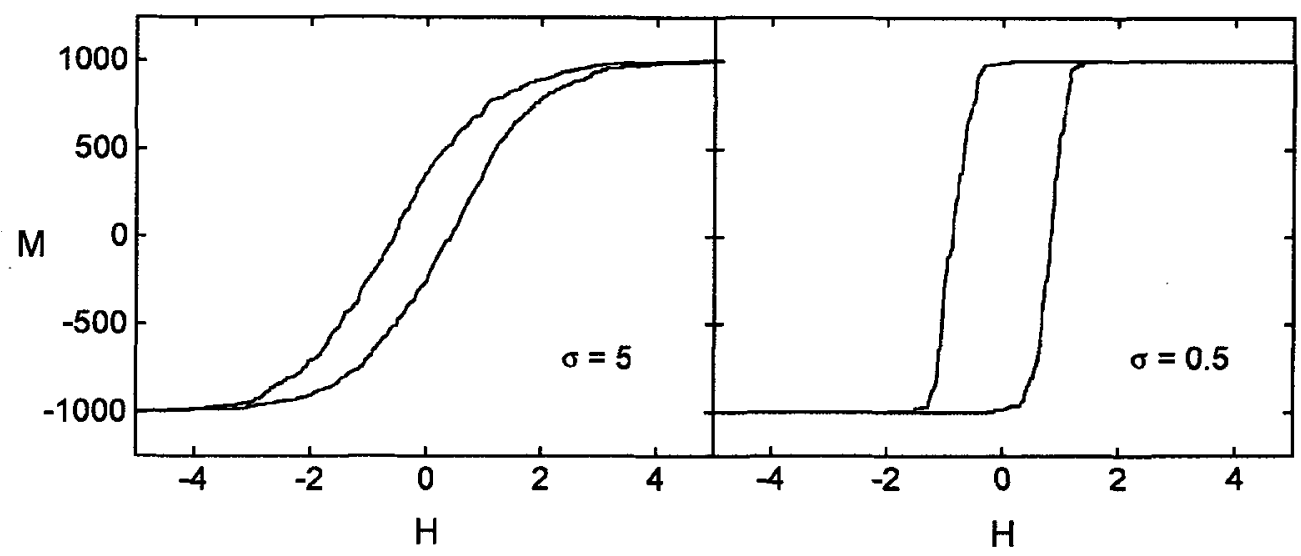

Figure 3: Shown is the effect of $\sigma$ (the width of the distribution of random fields $\left\{h_{i}\right\}$ ) on the shape and extension of the hysteresis cycle of a RFIM with $d=1$ and $N=1000$ spins.

and thus, on its turn, comes from two separate contributions: the interaction energy between neighbouring spins, and the interaction energy with the quenched random fields. The potential $\mathcal{F}$ is function only of the configuration $\left\{s_{i}\right\}$. It is a true state function, since, according to Eqs.(15) and (21):

$$
\oint d \mathcal{F}=\oint H d M-\oint T \vec{d} S_{i}=0
$$

In conclusion, our thermodynamic analysis has identified and separated the different contributions to the energy of the system in each transformation step. These results are relevant, since relations of this kind had been stablished up to now only for global changes in the overall hysteresis cycle.

The role of temperature deserves a comment. From a microscopic point of view we have assumed that the system is at $T=0$, to indicate that the system does not experience thermal fluctuations that can be considered relevant for the dynamics. Consequently, the dynamics of the system is completely deterministic and the transformation trajectories in configuration space perfectly reproducible from cycle to cycle. From a macroscopic (thermodynamic) point of view, the model represents a system that behaves in the way described regarding the dynamics, but is at a constant temperature $T$. Note that $T$ enters only as $T \vec{d} S_{i}$ in the thermodynamic analysis of the RFIM. The reason is that the two phases $(+1$ and -1$)$ are thermodinamically equivalent: they have the same entropy. We could equally well redefine $T \vec{d} S_{i}$ as a dissipated energy and totally ignore the role of temperature.

It is interesting to notice that the energy dissipated in the avalanches (Eq.(18)) and the magnetization jump at these same avalanches are not proportional, as demonstrated in Figure 2. In the energy dissipated, each contribution to the magnetization jump (arising from a flipping spin) is weighted by the local magnetic field of the spin (which depends on the configuration of neighbouring spins). Another interesting aspect of the model lies on the fact that the sequence of configurations followed from $\{-1\}$ to $\{+1\}$, on increasing $H$, bears some resemblance (particularly at a local level) with the configuration followed from $\{+1\}$ to $\{-1\}$, on decreasing $H$. The effect is shown also in Figure 2. The similarities come from the presence of a static field $h_{i}$ on each latice site, while the differences are due to the interaction between spins.

Finally, note that the statistical properties of the distribution $\left\{h_{i}\right\}$ have an effect on the dynamics of the transition and the hysteresis cycle. When the width of the distribution, $\sigma$, is large enough, 
most neighbouring spins experience very different random fields: the spins flip in a nearly uncorrelated manner. The transformation trajectories are made of small jumps of $M$ and the hysteresis cycle extends in a wide range of $H$, as shown in Figure 3. On the other side, in this same Figure we see that spin flips are highly correlated for small $\sigma$ and, correspondingly, the transformation trajectories display large jumps of $M$ in a relatively narrow range of $H$. The passage from one kind of behaviour to the other upon varying $\sigma$ is a true continuous phase transition, which has been extensively studied in recent years, both analitically and numerically $[2,8]$.

\section{APPLICATION TO THERMOELASTIC MT. CONCLUSIONS.}

The macroscopic behaviour of the fluctuationless RFIM resembles in many aspects the behaviour of thermoelastic MT. It is attractive to think that the reasons for the similarity lie, at a microscopic level, on an equivalent structure of the underlying Hamiltonian and equivalent dynamics. If this is the case, our analysis of the thermodynamics and memory properties of the RFIM also apply to thermoelastic MT. Let us examine the difficulties to be expected.

The most important difficulty, possibly, lies on writing a functional form of the energy stored, $\mathcal{F}$, which depends on the configuration of the system. It remains to be explored how to define the two-phase configuration of a martensitic system in terms of a reduced number of relevant variables, say the volume fractions of the different martensite variants on each cell of a coarse-grained reticle. $\mathcal{F}$ should represent the energy arising from the mechanic (elastic) interaction between austenite and martensite domains, and between these domains and lattice defects (which, to a first approximation, can be supposed static). This kind of study, which belongs to the domain of micromechanics, has seen important advances in recent years [9].

It is interesting to notice that the analysis made for the RFIM shows that, if the configurations in the martensitic system can be defined in a way such that a partial ordering of configurations can be stablished, and $\mathcal{F}$ has a functional form adequate to preserve the partial ordering in monotonic excursions of the driving field, then the memory properties of partial transformation cycles will arise as a natural consequence.

Concerning the thermodynamics of thermoelastic MT, we should take into account that the term $H d M$ (the energy input by the external field) must be replaced now by a sum of two terms, $F d L+T d S$, representing the driving force of the MT [10]. The term $F d L$ is the energy put into the system as mechanical work by a force $F$ (here uniaxial) and $L$ the coupled elongation. The term $T d S$ arises as a result of the fact that the two phases (austenite and martensite) have different entropy. The system is controlled by two independent external fields: temperature and applied stress.

That the two phases have different entropy has another important consequence: each transformation event is accompanied by a local release or absorption of transformation latent heat. Consequently we are facing a problem of heat transport out of equilibrium, with its corresponding entropy production of thermal origin (nor magnetic or mechanical). Presumably, the dependence of the width of the hysteresis cycle on the rate at which the cycle is carried out, and on the thermal properties of the medium with which the system exchanges heat, arises from this source [11].

To conclude, the fluctuationless RFIM can be regarded as a simple model to study dynamic and thermodynamic properties of driven first-order phase transitions in the presence of disorder. The extension of this study to more complex real systems, particularly to thermoelastic MT, promises to be exciting and rewarding.

\section{Acknowledgements}

We are recognized to the members of the Scientific Committee for their invitation. Through the years, we have benefited from many interesting discussions on the thermodynamics of MT with sev- 
eral colleagues, particularly A. Planes and L. Delaey and, more recently, E. Vives and Ll. Carrillo. This work has received financial support from the CICyT (Spain), project MAT95-0504.

\section{References}

[1] See the review papers by Ortín J., Planes A., Acta. Met. 36, Overview 68 (1988) 1873; ibid. 37 (1989) 1433; Cahn J.W., Prog. Mater. Sci. 36 (1992) 149; Wollants P., Roos J.R., Delaey L., Prog. Mater. Sci. 37 (1993) 227; and references therein.

[2] Sethna J.P., Dahmen K., Kartha S., Krumhansl J.A., Roberts B.W., Shore J.D., Phys. Rev. Lett. 70 (1993) 3347; Dahmen K., Sethna J.P., Phys. Rev. Lett. 17 (1993) 3222; Sethna J.P., Dahmen K., Kartha S., Krumhansl J.A., Perkovic O,, Roberts B.W., Shore J.D., Phys. Rev. Lett. 72 (1994) 947.

[3] Huang K., Statistical Mechanics (John Wiley and Sons, New York, 1987).

[4] Vives E., Planes A., Phys. Rev. B 50 (1994) 3839.

[5] Jiles D.C., Atherton D.L., J. Appl. Phys. 55 (1984) 2115; Jiles D.C., Introduction to Magnetism and Magnetic Materials (Chapman and Hall, New York, 1991).

[6] Ortín J., J. Appl. Phys. 71 (1992) 1454, and references therein.

[7] Several examples are quoted in Mayergoyz I.D., Mathematical Models of Hysteresis (SpringerVerlag, New York, 1991). See also Goicoechea J., Ortín J., Phys. Rev. Lett. 72 (1994) 2203-2206.

[8] Vives E., Goicoechea J., Ortín J., Planes A., Phys. Rev. E 52 (1995) R5-R8.

[9] See the review papers by Fischer F.D., Berveiller M., Tanaka K., Oberaigner E.R., Arch appl. Mech. 64 (1994) 54-85; Ball J.M., James R.D., Arch. ration. Mech. Analysis 100 (1987) 13-52; Ball J.M., James R.D., Phil. Trans. R. Soc. Lond. A 338 (1992) 389-450.

[10] Bruinsma R., Aeppli G., Phys. Rev. Lett. 52 (1984) 1547-1550.

[11] Bruno O.P., Leo P.H., Reitich F., Phys. Rev. Lett. 74 (1995) 746-749, and references therein. 